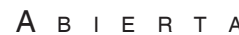

\title{
IMPACTOS DEL ZAPATISMO EN LA ESCUELA: ANÁLISIS DE LA DINÁMICA EDUCATIVA INDÍGENA EN CHIAPAS (1994-2004)
}

\author{
Raúl Gutiérrez Narváez
}

\begin{abstract}
La resistencia activa de los zapatistas a lo largo de estos años ha suscitado suspicacias, recelos y hasta críticas de "la izquierda" y el feroz encono del gobierno federal y de los poderes trasnacionales, pero nadie puede negar que es la expresión auténtica de las comunidades indígenas que buscan libremente su propio camino y, sobre todo, que han alcanzado logros notables, a pesar de los obstáculos que han enfrentado (Garrido, 2004).
\end{abstract}

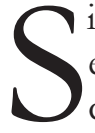
in duda, discutir sobre el impacto que ha tenido el zapatismo en la dinámica social, no sólo chiapaneca, sino mexicana e incluso mundial, se ha convertido en un asunto polémico; esto debido a que puede ser abordado -y de hecho, lo ha sido-desde diversas perspectivas. Con el objetivo de aportar elementos al debate que nos permitan valorar el impacto del movimiento zapatista, al menos en el ámbito de la educación formal; a continuación presento un análisis de las propuestas implementadas por diversos actores (no exclusivamente por el EZLN) en el campo de la educación indígena en Chiapas a partir del levantamiento armado.

Realizo este análisis con base en una etnografía del discurso público de diferentes agentes colectivos (organizaciones, instituciones) que actúan en el campo. ${ }^{1}$ De acuerdo con Gunther Dietz, estamos hablando de la dimensión semántica ("estrategias discursivas

Raúl Gutiérrez Narváez, egresado de la maestría en Antropología Social, CIESASSureste. empleadas por los diferentes actores pedagógicoinstitucionales") de la educación intercultural, tanto en el nivel de los enfoques y modelos elaborados y promovidos desde los discursos académicos, políticos y escolares, como en el nivel de "la concreción semántica de estos modelos a través de las didácticas y los diseños curriculares específicamente creados para responder al 'problema escolar' de la diversidad cultural" (Dietz, 2003: 157), incluyendo las auto y heterorrepresentaciones de los agentes hacia el exterior, plasmada en principios, planes y programas, declaraciones, informes, etc., y sin obviar el contexto social en el que se emiten estos discursos; es decir, la dimensión sintáctica de las estructuraciones institucionales (Dietz, 2003).

\section{Antecedentes}

Antes de 1994, la dinámica de la educación indígena en Chiapas, al igual que en todo el país, se caracterizaba por la inercia del modelo bilingïe-bicultural, ${ }^{2}$ que arrastraba 
hasta nuestros días las ideas asimilacionistas de principios del siglo xx. Aunque con un sinnúmero de etiquetas: asimilación, incorporación, integración, aculturación, bilingüe o bilingüe-bicultural, y los cambios de forma, que las han justificado; la educación indígena en México no había experimentado realmente, al menos al nivel de la práctica, cambios de fondo; sino más bien, había ido modificando su discurso, adaptándolo a las circunstancias políticas y académicas coyunturales y a las formas de resistencia de los pueblos indígenas (que durante muchos años se negaron a enviar a sus hijos a la escuela), pero sin abandonar realmente sus pretensiones —ortodoxamente liberales_ asimilacionistas y de homogeneización cultural. ${ }^{3}$

En su Primera Declaración de la Selva Lacandona, el EZLN incluyó a la educación como una de sus demandas centrales. Con este solo hecho, y a pesar de que no hicieron ninguna precisión sobre el tipo de educación que demandaban, los zapatistas dieron un gran impulso a las acciones en materia de educación indígena, inaugurando un periodo muy fecundo en análisis, propuestas, debates y prácticas pedagógicas innovadoras que, once años después, continúa.

La otrora esfera de acción exclusivamente gubernamental recibió el importante aporte de otros actores, principalmente de los mismos indígenas, quienes emprendieron acciones en varias direcciones, en función de sus trayectorias, intereses y posicionamientos, implementando novedosas propuestas alternativas al tradicional modelo de educación bilingüe-bicultural.

Para efectos de análisis he clasificado las diferentes propuestas educativas en tres grupos, en función del tipo de agente que las ha instrumentado: Oficiales, por parte de los gobiernos federal y estatal; Autónomos, por instancias del EZLN como municipios autónomos o Juntas de Buen Gobierno, que no cuentan con reconocimiento oficial e Independientes, por Organizaciones no gubernamentales, en coordinación con individuos o agrupaciones indígenas y religiosas (véase cuadro 1). ${ }^{4}$

\section{Educación bilingüe-comunitaria: ruptura y continuidad}

En una primera etapa, que podríamos ubicar entre 1994 y 1996, se implementaron varios proyectos educativos (sin la participación directa del EZLN) que, en términos generales, reprodujeron algunas estrategias recurrentes y con una larga tradición en la historia de la educación en México (presentes ya desde los inicios de la educación bilingüe, en los tiempos del indigenismo y luego implementadas por el Consejo Nacional de Fomento Educativo-CONAFE en su modalidad de educación comunitaria). Me refiero específicamente a una orientación técnico-comunitaria y al trabajo con jóvenes originarios de las comunidades, brevemente capacitados para la docencia.

Las primeras acciones provinieron directamente del gobierno federal. Dada la estrecha correspondencia entre la demanda zapatista y las políticas de ampliación de la cobertura educativa impulsadas por el Estado mexicano, como parte de un programa de "inversión" en capital humano promovido desde organismos internacionales (acorde con los planteamientos liberales), los reclamos zapatistas bien podían interpretarse como el fin de las resistencias indígenas a la educación escolarizada, por lo que encontraron una respuesta casi inmediata entre las instancias oficiales. El 28 de enero de 1994, la Secretaría de Educación Pública anunciaba "un Programa para Extender y Mejorar los Servicios Educativos en Poblaciones Indígenas, cuya primera fase se emprenderá en Chiapas, Guerrero, Oaxaca, Puebla, Hidalgo y Veracruz". ${ }^{5}$

Debido a las fuertes críticas que se hicieron a la modalidad de educación bilingüe, a los problemas que los maestros de este sistema enfrentaron en las comunidades indígenas, principalmente en la "zona de conflicto", y a que desde dos años atrás estaba a cargo del gobierno estatal, las acciones federales se concentraron en ampliar la oferta educativa a través de la 
E $R \quad$ T

modalidad desarrollada por el CONAFE, adaptando su propuesta de educación comunitaria al medio indígena. En un corto periodo, la cobertura educativa bajo esta modalidad se incrementó significativamente en el estado de Chiapas, pasando de 10863 alumnos atendidos en el ciclo escolar 1993-1994 a 29008 en el ciclo 19941995 (SEP 1995: anexo 1).

Dado que los docentes contratados son originarios y hablan la lengua de las comunidades en las que laboran, se afirma que imparten una educación bilingüe y CONAFE la ha catalogado como educación comunitaria indígena pero, aunque se plantean novedosas metodologías participativas, se trabaja conforme a los planes y programas, organización escolar y calendario de estudio nacionales.

De acuerdo con Enrique Pieck, la educación comunitaria surge en la década de 1970 impulsada por la UNESCO para "combatir los problemas derivados de las necesidades estructurales de los países en desarrollo", con una orientación hacia el desarrollo de la comunidad que, basada en

[...] una perspectiva funcionalista donde el desarrollo, la integración y la modernización de las comunidades se conciben como soluciones adecuadas para resolver los problemas de éstas [...], parte del presupuesto de que la gente de las comunidades mostrará interés en involucrarse en actividades que respondan a sus "necesidades reales" y, en consecuencia, estarán motivados para efectuar cambios en sus comunidades (Pieck, 1996: 89, 121-122).

Aunque la educación comunitaria se implementa en los países desarrollados como una modalidad de educación no formal, en México es aplicada en el ámbito de la educación básica (tanto en los programas de CONAFE como, desde mediados del siglo pasado, en los del $\mathrm{INI}^{6}$ ) con el propósito de atender a niños de localidades pequeñas y aisladas (que no son atendidos por otras modalidades) sin incurrir en los altos costos que implica el sostenimiento de una escuela convencional, mediante la capacitación y empleo de jóvenes instructores comunitarios, contratados bajo la modalidad de servicio social con una exigua retribución económica.

También el gobierno del estado de Chiapas respondió a la demanda zapatista con una propuesta similar a la de CONAFE: el Proyecto del Educador Comunitario Indígena (PECI). Los orígenes del PECI se remontan a los tiempos en que se crea la Asociación Rural de Interés Colectivo (ARIC), que aglutinó a cientos de comunidades y decenas de miles de campesinos, principalmente de la Selva Lacandona:

En 1988, las comunidades indígenas en las Cañadas, preocupadas de la situación educativa imperante en la región, originada por la desatención de los programas oficiales de educación y la falta de propuestas educativas adecuadas a la realidad sociocultural y ambiental de la Selva, plantearon la necesidad de educadores comunitarios originarios de las mismas comunidades que en respeto a los valores y prácticas culturales, ofrecieran una alternativa que respondiera a dicha situación. De esta manera, cinco comunidades de la Selva nombraron a sus propios educadores y pronto se sembró en el corazón de las demás comunidades esta misma inquietud (Lumaltik Nopteswanej, 2001: 6-7).

Así nació, de forma independiente y financiado con recursos del Banco Mundial, el Programa de Educación Integral de las Cañadas de la Selva Lacandona (PEICASEL); mismo que poco tiempo después sería reconocido, apropiado e integrado como un programa del gobierno del estado y para el año de 1990 sería ampliado a toda la Selva Lacandona bajo el nombre de Programa de Educación Básica de la Selva (PEBS). El Programa comenzó atendiendo una población de 960 alumnos en 1989 y llegó a contar a 7043 alumnos en el 
ciclo escolar 1993-1994 (Gobierno de Chiapas, 1993). Después del levantamiento zapatista, las escuelas del PEBS establecidas en comunidades mestizas fueron absorbidas por CONAFE y las establecidas en comunidades indígenas fueron paulatinamente integradas al nuevo PECI.

Con el PECI, el gobierno del estado de Chiapas incursionó por primera vez en el terreno de la educación indígena (identificada como tal por el origen y la lengua de los docentes), con el objetivo de ampliar la cobertura de los servicios de educación básica. El Proyecto plantea una propuesta educativa comunitaria, perspectiva que se concreta en la facultad que otorga a la comunidad para elegir al "elemento central del proyecto": el Educador Comunitario Indígena, aunque se abroga la facultad de definir "las políticas educativas, las metas, los tiempos y las modalidades que habrán de adoptarse para la ejecución del programa por parte de las comunidades" (Secretaría de Educación, 1996), con base en los programas vigentes a nivel nacional.

El proyecto creció considerablemente en los primeros años, estableciendo escuelas en comunidades que antaño carecían de servicios educativos, incluso llegó a instalar centros escolares en comunidades que ya contaban con otro tipo de servicio (sea bilingüe federal o monolingüe estatal) pero que habían manifestado inconformidad con el mismo, y hasta en zonas eminentemente zapatistas en las que se habían cerrado las escuelas a raíz del levantamiento armado. En el ciclo escolar 1994-1995 el PECI atendió a 2800 niños y para el año siguiente a 10500 alumnos.

En términos generales, la propuesta de PECI es muy similar a la de CONAFE, ambas tienen como objetivo principal ampliar la cobertura de la educación básica, adaptan sus experiencias comunitarias para responder a las demandas de educación indígena en poblaciones pequeñas y marginadas, contratando a jóvenes indígenas al margen de las normas laborales e introduciendo el uso de la lengua materna en la escuela, pero rigiéndose por los planes y programas nacionales.

Las acciones educativas no provinieron exclusivamente de las agencias estatales. Varios grupos de indígenas, coordinados con varias ONG, grupos religiosos y diversas instituciones, aprovecharon la coyuntura generada por el alzamiento zapatista e implementaron proyectos educativos que, aunque sustentados en múltiples antecedentes, integraron elementos similares.

En 1994, habitantes de la comunidad indígena de Guaquitepeq, municipio de Chilón, comenzaron a realizar los trámites para la apertura de una secundaria en ese lugar. ${ }^{7}$ Con la intermediación de religiosos jesuitas y el apoyo de la ONG "Patronato Pro Mexicano, A.C.", quien se encargó de gestionar los permisos oficiales y los financiamientos a través de fundaciones internacionales, en 1995 abrió sus puertas la escuela secundaria particular "Emiliano Zapata"; efectuando un modelo de educación bilingüe-bicultural adaptado al contexto local, desarrollado por el Centro de Desarrollo Rural (CESDER, otra ONG), con base en su experiencia en la tele-secundaria de Yahuitlalpan, municipio de Zautla, Puebla, desde 1989. El modelo se basa en la articulación de los contenidos del programa nacional con la capacitación sobre cuestiones agropecuarias y talleres técnicos y de investigación (Méndez, 2000).

La escuela comenzó siendo atendida por cuatro profesionales mestizos venidos del estado de Puebla, a los que posteriormente se sumaron dos traductores de la comunidad para superar las dificultades de comunicación con los alumnos. Al mismo tiempo, la Secretaría de Educación, a solicitud de "los ladinos e indígenas priistas de la comunidad", quienes veían estas acciones como una amenaza, abrió una escuela telesecundaria, cuya apertura se había estado gestionando, sin éxito, desde varios años atrás (Méndez, 2000: 43). 
A B I E R T A

También en el año de 1995, indígenas interesados en la educación (organizados a partir de un curso de capacitación de PECI) y comisionados por sus respectivas comunidades, solicitaron la asesoría de académicos del CIESAS y crearon la Unión de Maestros de la Nueva Educación para México (UNEM), con el objetivo de trabajar una propuesta alternativa de educación primaria e "[...] implementar una profunda reforma del proceso educativo básico que combina la teoría y la práctica bajo el control de las comunidades indígenas de Chiapas [...]" (UNEM, 1999: 4).

Con el apoyo de CIESAS y DANA, A.C., UNEM esta unión organizó una serie de cursos iniciales sobre el manejo de la agricultura orgánica en la huerta escolar, durante los últimos meses de 1995 y el primer semestre de 1996, con la participación de cerca de un centenar de jóvenes educadores indígenas de las zonas Altos, Norte y Selva. Los cursos fueron creados con base en las experiencias previas sobre agricultura orgánica de DANA, tras el desarrollo de diferentes proyectos de agroecología en otros estados del país. A mediados de 1996 se comenzó a trabajar con una nueva propuesta pedagógica para la educación primaria que organiza los contenidos y las prácticas escolares a partir de las actividades cotidianas en las que los niños participan, tanto en la comunidad como en la huerta agroecológica, y se implementaron tanto en escuelas con registro oficial (principalmente de PECI) como en escuelas autónomas como el caso de la comunidad de La Pimienta en el municipio de Simojovel (Gutiérrez, 2005).

También en 1995, con la coordinación entre los habitantes de una comunidad zapatista y la ONG Enlace Civil, nace el que a la larga se ha convertido en el principal proyecto educativo zapatista: Semillita del Sol, en un principio dedicado únicamente a la enseñanza de artes manuales:

En abril de 1995, en una comunidad de la zona selva tojolabal se comienzan los trabajos del proyecto de
Semillita del Sol con la participación de 40 niños de dicha comunidad que comienzan a tomar clases de artes manuales por un periodo de seis meses. Posteriormente la comunidad plantea la necesidad de la enseñanza primaria para los niños que hasta 1995 nunca habían tenido una enseñanza formal y es cuando se empieza a planear el programa de educación primaria y alfabetización basada en los usos y costumbres de las comunidades indígenas en Chiapas (Enlace, s.a.).

\section{Autonomía e interculturalidad: los Acuerdos de San Andrés en materia educativa}

Entre tanto, la dinámica político-militar del conflicto zapatista desembocó en los diálogos de San Andrés entre el gobierno federal y el EZLN, que incluyeron como uno de los temas de discusión la "Promoción de la educación bilingüe e intercultural", anticipando desde el principio, algunas de sus conclusiones. Por primera vez los zapatistas se manifestaron por una educación diferenciada, que respondiera a las características socioculturales de los grupos a los que iba dirigida, $y$ dejaron en claro que no demandaban únicamente la ampliación de la cobertura educativa; que además de acciones cuantitativas exigían al gobierno cambios cualitativos en el ámbito de la educación indígena y, sin argumentos en contra, los negociadores del gobierno firmaron una serie de acuerdos que perfilaban cambios importantes.

Los acuerdos que en materia educativa se alcanzaron en San Andrés reflejan la diversidad de los actores que participaron en las negociaciones (maestros y activistas indígenas, científicos, políticos y funcionarios entre otros), así como, paradójicamente, la falta de consenso en torno al tema, y van desde la simple reiteración de ampliación de la cobertura de los servicios educativos hacia los grupos indígenas hasta la participación 
fundamental de los mismos en el rediseño de las políticas educativas en general, poniendo el acento en dos aspectos centrales: su carácter intercultural y autonómico, aunque sin precisar exactamente lo que se entiende por ellos.

1. Del discurso liberal al discurso multicultural: la etnificación de las demandas educativas

La reorientación de las demandas educativas del EZLN, al pasar de "educación completa y gratuita" en 1994, a educación autónoma e intercultural, no puede entenderse al margen de la estrategia con que los zapatistas enfrentaron el diálogo con el gobierno mexicano. Con el objetivo de "abrir" el espacio de lucha a otros sectores de la "sociedad civil" y darle carácter nacional al movimiento, como respuesta a la pretensión gubernamental de reducirlo al nivel local, el EZLN —al igual que el gobierno federal— contó con la participación de personalidades y expertos en cada uno de los temas discutidos, en calidad de invitados y asesores.

Un intenso diálogo con diversos movimientos sociales y culturales llevó al EZLN a acercarse a lo que Dietz (2003) llama el multiculturalismo y a retomar algunos de sus planteamientos, entre ellos la educación intercultural o pluricultural. El origen de la educación intercultural puede situarse en el marco de la práctica educativa surgida como resultado de los movimientos de reivindicación étnica que se han presentado, desde la década de 1960, en muchos Estados-nación "occidentales" (entre ellos, México) y que reflejan el fracaso relativo de las políticas educativas homogeneizantes. Estos movimientos han surgido en el marco de los procesos de globalización, característicos de la etapa neoliberal del sistema capitalista, que han intensificado los flujos internacionales de capitales, mercancías, símbolos y personas y que han implicado nuevos patrones de interacción cultural entre, en un extremo, la pretensión de las empresas trasnacionales de universalizar los patrones de consumo y las pautas de conducta de los países altamente industrializados como medio para expandir sus mercados y, en el otro, la generación, como respuesta, de una serie de procesos locales que van desde la asimilación, pasando por la adaptación hasta la resistencia y la reivindicación de las diferencias culturales. Son un reflejo también del enorme potencial político transformador que han adquirido las diferencias culturales:

\footnotetext{
La cultura es un ámbito de la vida sobre el cual los grupos subordinados suelen mantener algún control. Precisamente por ello puede ofrecer un reducto del que los grupos subordinados nutren su disidencia moral contra la dominación (Dietz, 2003: 27).
}

Las respuestas ofrecidas por los Estados liberales han variado en función de la fuerza de los movimientos y han ido desde políticas bilingües (Suiza, Bélgica, Holanda, Malta, Canadá, Finlandia, Bolivia), programas escolares bilingües, de transición (varios países latinoamericanos), hasta la concesión de grados diversos de autonomía (España, Alemania, Nicaragua). Como afirma Gunther Dietz, "el modelo prototípico de conceder 'derechos históricos' a determinados grupos subnacionales ha sido desarrollado para aquellos casos en los cuales el movimiento étnico-nacional contrahegemónico amenazaba con fragmentar el conjunto del Estado-nación" (Dietz, 2003: 119).

Esa misma fuerza ha influido para que el debate sea retomado por diversos teóricos del liberalismo, que han reconocido el pluralismo cultural de las sociedades contemporáneas y han formulado mecanismos de negociación e integración de esta diversidad en los marcos liberales como la propuesta de Kymlicka (1996) de una ciudadanía multicultural, llevando el debate hacia un terreno marcadamente reformista, hacia la liberalización del multiculturalismo. 
Es así como, a finales de la década de 1980, el discurso multiculturalista es retomado por organismos internacionales, despojado de su connotación política radical e impulsado en diversos foros como parte de una nueva política global de respeto a las diferencias. En 1989 dicho discurso fue incorporado en el Convenio 169 de la OIT, que sería signado por numerosos países, entre ellos México en 1990 y que incidió en las reformas al artículo $4^{\circ}$ constitucional, realizadas en 1992, en las que se reconoce el carácter pluricultural de la nación mexicana. En la misma dirección, en 1990, la Dirección General de Educación Indígena adoptó, nominalmente ${ }^{10}$, la modalidad de educación intercultural-bilingüe (sustituyendo el modelo bilingüe-bicultural, vigente desde 1978).

Este multiculturalismo neoliberal, light, gestionado o corporativo, entre otras etiquetas que le han sido impuestas, ha sido fuertemente cuestionado por algunos autores como Charles Hale, quien ha afirmado que "el proyecto cultural del neoliberalismo consiste en domesticar y redirigir la abundante energía política que muestra el activismo de los derechos culturales, en vez de oponerse directamente a dicho activismo" (Hale, 2002: 10); conformándose una corriente radicalizada, el multiculturalismo emancipatorio, crítico, revolucionario o pluralista, que pugna por un rescate del carácter transformador del discurso multicultural: "el multiculturalismo revolucionario no se limita a transformar la discriminación mediante actitudes, sino que se dedica a reconstituir las estructuras profundas de la economía política, la cultura y el poder en los arreglos sociales contemporáneos" (McClaren, 1998: 289).

Así es como, dada la diversidad de experiencias a las que alude, reflejo del intenso campo de fuerzas en el que se ha desarrollado, se carece de una definición común de lo que se entiende por educación intercultural. De hecho, bajo la misma denominación se han incluido una serie de políticas y prácticas muy diversas y hasta antagónicas, que van desde programas compensatorios, cursos, seminarios o muestras de folcklore paralelos al programa formal, inserción de contenidos étnicos en el currículo, incluso en forma de áreas o disciplinas; utilización de la lengua de los grupos minoritarios como lengua enseñada y como lengua de enseñanza; empleo de docentes del grupo minoritario, adecuación de la enseñanza a las características y necesidades de los diferentes grupos culturales, promoción del pluralismo cultural, hasta, al menos en el discurso, la interacción en términos de igualdad de las diferentes culturas en el aula. En la mayoría de los casos, estas acciones se han restringido al ámbito de la educación dirigida a grupos indígenas.

De esta forma, el concepto de educación intercultural abarcaría a todas aquellas prácticas educativas que tratan de responder a la diversidad cultural de las sociedades actuales, con muy variados objetivos en un marco de desigualdad social y de conflicto intercultural. ${ }^{11}$

La cultura, y la concepción que de ella se tiene, juega un papel central en la definición de los diferentes proyectos de educación intercultural y en la mayoría de ellos se hallan implícitas concepciones estáticas y esencializadas de la cultura desde hace mucho tiempo superadas en el análisis antropológico. ${ }^{12}$ Sin embargo, es preciso reconocer que ciertos "patrones de interacción comunicativa y simbólica" o "formas de vida" (cultura en términos de Peter McClaren) o "prácticas habitualizadas" (en términos de Gunther Dietz) pueden ser explícitamente identificados como propios por ciertos grupos a través de procesos de etnificación cultural que se desencadenan en situaciones de contacto con otros grupos, en las que surge la necesidad de autoidentificarse y de diferenciarse de los Otros, y en los que entran en función diversas representaciones, intereses y relaciones de poder; de lo que resulta la reorganización, resignificación y renormativización de lo que es considerado como propio y ajeno; procesos de autonomía y hetero- 
nomía, que imprimen su sello en las propuestas educativas interculturales.

\section{Un marco autonómico para la práctica educativa}

De la misma forma que con la interculturalidad, los planteamientos autonómicos de los zapatistas se nutrieron de las experiencias y propuestas de sus invitados y asesores, de lo establecido en el Convenio 169 de la OIT y, además, de una larga tradición de vida autónoma, al margen del Estado nacional, desarrollada por los pueblos indígenas mesoamericanos, como parte de sus estrategias de resistencia:

En la práctica, muchos pueblos indios han disfrutado algún grado de autonomía de esta naturaleza, en diferentes momentos históricos. Sin embargo, dado que ese género de 'autonomía' resulta de la permisividad del poder o de la imposibilidad de éste de imponer plenamente su voluntad en un momento dado, más temprano que tarde — una vez que han cambiado las condiciones originales_- las mercedes o gracias se ven troncadas por la misma voluntad que las concedió (Díaz Polanco, 2003: 151).

Aunque fue el tema específico de una de las mesas, éste se trató ampliamente en todas las restantes, dado que se había convertido en el eje del planteamiento zapatista, y ya formaba parte de su discurso desde principios de 1994:

Autonomía, dicen los compañeros, como la de los vascos, o la catalana, que es una autonomía relativa [...] Pues entonces ellos dicen que hay que negociar un estatuto de autonomía donde nuestro gobierno, nuestra estructura administrativa, sea reconocida por el gobierno y podamos convivir así, sin que se metan con nosotros. ${ }^{13}$
Aunque los contenidos de las propuestas autonómicas zapatistas fueron "sustancialmente recortados", al grado de convertirlos —en palabras de Héctor Díaz Polanco (2003) _ en un planteamiento "pseudoautonómico", aparecieron como parte de los acuerdos alcanzados. De esta forma se garantizaba al menos un mínimo marco autonómico en el que se pudiera desarrollar la práctica educativa intercultural, acorde con los planteamientos de Freire (1994), quien considera que es imperativo otorgar autonomía a las escuelas, pero no una autonomía que implique la huida del Estado de su deber de ofrecer educación de calidad y en cantidad suficiente para atender la demanda social. Esta autonomía implica el derecho de los actores locales, de los grupos indígenas, a la autodeterminación en materia educativa en sus más diversos aspectos: desde las formas de organización escolar, hasta la definición de planes y programas de estudio y la conformación de sus propios sistemas educativos.

\section{E1 in-cumplimiento de los Acuerdos}

Los Acuerdos de San Andrés fueron firmados en un ya célebre acto protocolario el 16 de febrero de 1996. Su firma despertó muchas expectativas y especulaciones sobre la inminente solución al conflicto zapatista. Sin embargo, poco se avanzó en la concreción de los mismos, el diálogo fue suspendido y el conflicto se ha prolongado hasta nuestros días. Con el Ejército Zapatista dominado en términos militares ${ }^{14} \mathrm{y}$ con la división que comenzó a gestarse entre sus grupos de apoyo, ${ }^{15}$ la movilización generada en respaldo de los Acuerdos de San Andrés ya no fue capaz de amenazar seriamente la estabilidad de los grupos hegemónicos ni de hacer avanzar la agenda pactada.

En este escenario se gestó la negativa del ejecutivo y legislativo federales a aprobar la iniciativa de ley que elevaría a rango constitucional los Acuerdos de San 
Andrés. El punto central de la controversia ha sido el de la autonomía de los pueblos indígenas, tajantemente rechazado por los políticos neoliberales y neoconservadores actuando desde el Estado, porque por su fondo, más que por su forma, contradice los valores neoliberales de la propiedad privada y del libre mercado.

A pesar de ello, muchas acciones se continuaron realizando, y muchas más se comenzaron a realizar, ahora inspiradas en los Acuerdos de San Andrés. Aquí podemos identificar el inicio de una nueva etapa, ahora caracterizada por la acción directa del EZLN en el terreno educativo, integrando en sus propuestas tanto interculturalidad como autonomía (principalmente esta última), y la reorientación, en el mismo sentido, de los proyectos independientes; mientras que el Estado concentraría sus acciones en programas compensatorios y de ampliación de la cobertura a nivel medio y medio superior.

En el mismo año de 1996, y sin hacer mención de los acuerdos de San Andrés, el PECI, en su propuesta de reorientaciones 1997, “[...] reconoce como fundamental la necesidad de ubicar conceptualmente al proyecto en la posición de interculturalidad", reorienta sus objetivos y se propone "desarrollar una alternativa de educación bilingüe intercultural en los niveles de preescolar y primaria cuya operatividad se derive de las expectativas y necesidades de las comunidades indígenas" (Secretaría de Educación, 1996), pero ajustándose a los planes y programas nacionales.

También en 1996, el Patronato Pro-Mexicano, A.C., "decidió realizar una acción paralela al trabajo de la secundaria, con la finalidad de capacitar y calificar a los jóvenes indígenas que atienden la escuela como educadores rurales bilingües-interculturales" y echó a andar el programa de técnicos en educación rural bilingüe intercultural nivel bachillerato, en la misma comunidad de Guaquitepeq, bajo el régimen de educación particular. La nueva propuesta divide en cinco grupos los componentes del universo temático a trabajar: la capacitación en el manejo de procesos educativos para la dignidad, la identidad y la autonomía; la interculturalidad como definición y aclaración de lo propio; la educación y producción para la subsistencia; la formación para la docencia en el nivel de educación básica; y los contenidos del nivel y conocimientos reforzadores (Berlanga, 1996), y también fue incorporada en la escuela secundaria. ${ }^{16}$

UNEM también reorientó su proyecto. Con el reiterado apoyo de CIESAS, DANA, la Comisión Nacional de Derechos Humanos y otras organizaciones, en 1997 inicia los cursos del "Bachillerato pedagógico comunitario para la formación de docentes de educación primaria bilingüe intercultural" en modalidad semiabierta y sin reconocimiento oficial. Su propuesta incluye la implementación de una pedagogía basada en un proceso dialógico y autogestivo de la educación, el rescate de los elementos y la expresión de la cultura e idiomas locales como base de la enseñanza preescolar y primaria y la enseñanza de las ciencias naturales y agroecología basado en la apropiación tradicional y el conocimiento local de la naturaleza (UNEM, 1999).

\section{La educación zapatista: la autonomía como base de la interculturalidad}

En 1997, el EZLN decide modificar su estrategia, abandona el diálogo y se declara en resistencia ante las instancias gubernamentales ${ }^{17}$ descartando cualquier tipo de negociación y rechazando los apoyos provenientes de las mismas, y anuncia una serie de acciones encaminadas hacia el fortalecimiento de la autonomía en sus territorios, redefiniendo los municipios rebeldes como autónomos y creando toda una estructura paralela a los ayuntamientos constitucionales:

En estas condiciones, las comunidades en resistencia tomaron la iniciativa de formar su propia organización 
educativa, como parte del proceso de construcción de la autonomía. Por eso han resistido las ofertas educativas del gobierno, que ni los toman en cuenta ni resuelven a fondo los problemas (Enlace s.a.:2).

A partir de este momento se pusieron en marcha varios proyectos educativos zapatistas que, aunque sin comunicación directa entre ellos, adoptaron algunas características similares, como el trabajo con jóvenes indígenas, con estudios de primaria o secundaria, comisionados por sus respectivas comunidades para desempeñarse como promotores de educación, quienes recibieron el apoyo solidario de la comunidad, sea en especie o en trabajo en su parcela, para su sostenimiento, son capacitados en las mismas comunidades por profesionistas simpatizantes del movimiento, venidos de la ciudad (regularmente también jóvenes) y construyen sus propuestas educativas de forma colectiva, buscando articular elementos de la cultura indígena con contenidos "nacionales" y universales y con los principios zapatistas, e integrando en el proceso a diversos actores de la comunidad. Cabe aclarar que estos proyectos no cuentan con reconocimiento de validez oficial.

En la región Cañadas de la Selva Lacandona comienzan los trabajos del proyecto de educación autónoma "Semillita del Sol", cuyos cursos de capacitación de promotores de educación dan inicio en julio de 1997. El proyecto propone un modelo educativo comunitario y pone el acento en su carácter autónomo:

La escuela que deseamos no puede ser una institución ajena a la comunidad, a la cual da servicio; debe ser mas bien, parte integral de la comunidad, debe pertenecer a ella en sentido pleno. Ello conlleva la participación de la comunidad en la concepción de la escuela, en las decisiones que atañen al conjunto del proceso formativo (desde los contenidos y las formas de la enseñanza, las cuestiones operativas -horarios, calendarios, recursos-, hasta el sentido de la escuela en tanto espacio de generación de saberes colectivos). La escuela tendrá que subordinarse a la decisión del colectivo al cual pertenece, es decir, a la comunidad (Enlace s.a.: 8-9).

Se trabaja con base en la integración de diferentes conceptos alrededor de un tema o problema en común, así como con la integración de "saberes locales y escolares en los espacios de Historias (en plural), Matemáticas y Vida y Medio Ambiente" (Enlace s.a.: 6).

En este proyecto, la definición de los contenidos propios en el aprendizaje se plantea a partir de tres vertientes: la revaloración de los saberes locales, la valoración de conocimientos que provienen de fuera de una localidad dada y la reflexión en cuanto a lo deseado, lo útil y lo necesario (Enlace s.a.: 7).

Semillita del Sol comenzó a trabajar en comunidades que no contaban con servicios educativos. En los años posteriores, el proyecto ha tenido un importante crecimiento y desplazó a los maestros oficiales que aún continuaban trabajando en comunidades zapatistas. Actualmente se encuentra funcionando en varias regiones zapatistas, en la zona Selva y en la zona Norte. Aunque no existen datos precisos sobre la población atendida, en julio del año 2000, el Director de Educación Elemental del gobierno del estado de Chiapas declaraba que

[...] unos mil alumnos se graduaron en las escuelas autónomas zapatistas al término del actual ciclo escolar. En un primer momento, en al menos 80 escuelas de la zona de influencia zapatista se inició el proceso educativo paralelo. Los mentores zapatistas manejan un servicio educativo alterno, aunque no tienen el reconocimiento, ni lo tendrán, por parte del Sistema Educativo Nacional, porque no estuvieron bajo 
nuestra supervisión [...] (Global Report, no. 78, 13-

19 julio del 2000, www.agrnews.org).

También en comunidades zapatistas de los Altos de Chiapas se echaron a andar proyectos de educación primaria autónoma con características similares. Además, desde 1997 comienzan los trabajos para la creación de la Escuela Secundaria Rebelde Autónoma Zapatista Primero de Enero (ESRAZ) en el Aguascalientes de Oventik, municipio de San Andrés que, con el apoyo económico de la organización "Schools for Chiapas", con sede en San Diego, California, comienza sus cursos de capacitación el 12 de diciembre de 1998.

La escuela abrió sus puertas a 180 alumnos provenientes de municipios autónomos aledaños al Aguascalientes el 10 de abril del año 2000, funcionando bajo la modalidad de albergue. La propuesta de la escuela pone énfasis en el carácter autónomo y liberador de la educación, sustentando su trabajo en la pedagogía liberadora de Paulo Freire, organiza los contenidos en varias áreas temáticas: Comunicación, Matemáticas, Ciencias Sociales, Ciencias Naturales, Humanismo, Producción y Tsotsil y plantea partir del conocimiento local hacia el conocimiento global a través de preguntas o actividades generadoras (véase Muñoz Ramírez, 2004).

En 1998, comienza sus trabajos otro proyecto de educación primaria en el municipio autónomo de Polhó, en los Altos de Chiapas, éste con el apoyo de la asociación civil Ta spol be (abriendo camino), creada específicamente para ello. El proyecto acondicionó varias pequeñas escuelas en cada uno de los diferentes campamentos instalados en Polhó para atender a los niños desplazados por los acontecimientos suscitados en la región durante el año de 1997 que terminaron con la tristemente célebre masacre de Acteal.

El proyecto define su propuesta educativa como intercultural, participativa y liberadora y divide los contenidos en siete áreas de conocimiento: Lengua Materna, la Castilla como segunda lengua, Matemáticas,
Entorno Natural, Entorno Social, Identidad y Zapatismo (véase www. bigfoot.com/taspolbe).

En 1997, la ARIC, en cuyo seno había surgido el PEICASEL, antecedente de PECI y de unEM, y que desde 1994 había sufrido una serie de divisiones derivadas de su interacción al nivel de la cotidianeidad con el EZLN, se reorganiza y

[...] el 22 de diciembre de 1997, la Asamblea General de Delegados de la ARIC Unión de Uniones-Coalición de Organizaciones Autónomas de Ocosingo, acuerda la salida de los instructores del CONAFE y de los maestros bilingües de la SECH y el nombramiento de educadores comunitarios. Son 32 comunidades tzeltales de los municipios de Ocosingo y de Chilón las que se suman al desafío de construir una propuesta de educación primaria intercultural bilingüe (Lumaltik, 2001: 7).

Con la asesoría, gestión financiera y capacitación de Enlace, Comunicación y Capacitación, A.C., ${ }^{18}$ y con la participación del Municipio Autónomo Zapatista Primero de Enero, crean el Programa de Educación Comunitaria Indígena para el Desarrollo Autónomo (ECIDEA), mismo que ponen en práctica a partir del ciclo escolar 1998-1999, también sin reconocimiento de validez oficial, atendiendo a un total de 1200 niñ@s, provenientes de 25 comunidades en resistencia y un municipio autónomo zapatista. En el verano de 1999, los educadores indígenas del proyecto conforman la asociación civil Lumaltik Nopteswanej (educadores de nuestro pueblo). Este proyecto integra explícitamente las perspectivas comunitaria, intercultural y autónoma en su propósito general:

En el programa ECIDEA se propone una educación indígena intercultural bilingüe, basada en concepciones y estrategias de la educación popular y comunitaria, a fin de favorecer el desarrollo autónomo de las comunidades e individuos que lo integran. Las concepciones de aprendizaje se supeditan a estos modelos y 
finalidades, por lo cual se retoman las teorías del aprendizaje significativo, aprendizaje por descubrimiento y de la metacognición (Lumaltik, 2001: 40).

La matanza de Acteal dejó también varios cientos de desplazados de la Sociedad Civil Las Abejas, por lo que muchos niños quedaron sin atención educativa en los campamentos; ante lo cual, a partir de 1999, se comienza a trabajar en un proyecto educativo para atender esta demanda. La organización Las Abejas, simpatizante del EZLN, en coordinación con jesuitas de la Diócesis de San Cristóbal, echa a andar la Escuela Primaria Autónoma Tsotsil, que se estableció en las comunidades de Acteal, Xoyep y Yibeljoj, municipio de Chenalhó, atendiendo a aproximadamente 400 niños con el trabajo de 29 promotores, jóvenes indígenas elegidos en sus comunidades, impartiendo conocimientos sobre el Maíz, la Madre Tierra y la Familia entre otros (Klein, 2001).

Como bien podemos apreciar, en todas estas propuestas "zapatistas" están presentes ciertos aspectos de la educación comunitaria similares a los de CONAFE y PECI (que incluso encontramos desde los planteamientos de la educación indigenista de mediados del siglo pasado). Sin embargo, su orientación es diferente, hacia los modelos de educación popular comunitaria, que tienen en Paulo Freire a uno de sus principales impulsores y que, según Enrique Pieck, "[...] en lugar de enfatizar la integración en un sistema socioeconómico ya establecido se preocupa fundamentalmente por fortalecer (to empower) a la comunidad $[\ldots y]$ promueve una participación que apunta hacia la 'liberación' vinculada con la noción de clase social" (Pieck, 1996: 119), hacia la transformación de las estructuras de poder a través de la recuperación del conocimiento cotidiano como punto de referencia para valorar el conocimiento externo; enriqueciendo así, los modelos críticos de educación intercultural con su propuesta autonómica, que devuelve a los indígenas el derecho a educar a sus generaciones jóvenes desde su propia representación del mundo y de sí mismos.

\section{Más allá del Estado y del mercado: las ONG}

"Si se sale el Estado, entran las fuerzas del mercado y eso es peor", fue el comentario de una de las asesoras de UNEM en una reunión en la que proponían reconsiderar la relación del proyecto con el gobierno del estado de Chiapas. Ésta ha sido una de las cuestiones de continuo debate en el desarrollo de los diferentes proyectos: su relación con el Estado. Podemos afirmar que todos los proyectos educativos que han actuado al margen del Estado, sean independientes o autónomos, lo han hecho después de que no encontraron una respuesta satisfactoria a sus demandas por parte del mismo; ante lo que se acercaron a personas o instituciones “independientes" en busca de asesoría. Así fue como la mayor parte de ellos entabló algún tipo de relación con algunas $\mathrm{ONG}^{19}$ y se presentó lo que María Bertely (2003) llama el tránsito de la intermediación corporativa a la intermediación civil. El que éstas estuvieran allí o que posteriormente se conformaran como tales, no es un hecho aislado y fortuito, sino que es parte de los cambios estructurales que están viviendo las sociedades "occidentales" en la etapa de la globalización.

Este auge de las ONG tiene varias fuentes. Responde a las políticas neoliberales de adelgazamiento del Estado, mediante las que se pretende relegar sus obligaciones sociales en manos de la iniciativa privada, en las fuerzas del mercado, por lo que podría pensarse que el zapatismo simplemente le ha facilitado las cosas al gobierno mexicano. Además, como afirman Bresser y Cunill, "parte de las prácticas que desarrollan las ONG lo hacen no sólo para enfrentar déficit o vacíos dejados por el Estado o el mercado, sino a partir de un proyecto de contención de la influencia de ambos sobre la vida 
social" (Enlace, s.a.: 40). Éste sería más bien el caso de las ONG que nos ocupan. De forma que no sólo no están realizando la "tarea" por el Estado, sino que lo están haciendo contrariando sus preceptos y en función de un proyecto político contrahegemónico, que busca presionar para que el Estado asuma sus obligaciones en la materia pero respondiendo a los requerimientos de sus destinatarios; obviamente, no de manera homogénea y "definida", sino más bien, en un marco de relaciones complejo y contradictorio (dialéctico).

\section{Hegemonía y dominación: dinero y represión como respuesta del Estado}

Los gobiernos federal y estatal, por su parte, concentraron sus esfuerzos en impulsar programas de tipo compensatorio (promovidos desde el Banco Mundial y el Banco Interamericano de Desarrollo) y entre 1997 y 2000, como parte de toda una estrategia política para minar la base social del EZLN y contrarrestar sus acciones de resistencia, intensificaron el otorgamiento de exiguas becas a los alumnos indígenas a través del programa de educación, salud y alimentación (PROGRESA) que, junto con otros programas de becas preexistentes, COMO FIDUCAR de CONAFE o el de SECH, prácticamente han llegado a apoyar a la totalidad de los alumnos inscritos en las escuelas oficiales de las comunidades indígenas, a partir del tercer grado de primaria y hasta concluir los estudios de secundaria, e incluso de bachillerato, ahora bajo el nombre sexenal de Oportunidades.

Lo mismo ha sucedido con la expansión del programa de "desayunos escolares" que, con financiamiento federal, estatal y municipal, otorga una serie de alimentos industrializados a los niños para que los consuman al inicio de la jornada escolar. ${ }^{20}$ Estos programas compensatorios han constituido una de las respuestas "preferidas" de los gobiernos liberales para enfrentar la diversidad cultural (vista más bien, como diferenciación social) en las escuelas y están basados en la teoría funcionalista del capital humano:

En sentido amplio, se denomina política educativa compensatoria al conjunto de medidas políticas, económicas, sociales y escolares de discriminación positiva que se aplican sobre una población afectada por pobreza económica, social y cultural, en orden a reducir y paliar su desventaja en el sistema educativo (Muñoz Sedano, 2000: 82).

Otra parte de las acciones de los gobiernos se ha centrado en la ampliación de la cobertura, ahora de la educación media básica y media superior, con el establecimiento de un importante número de planteles de telesecundaria, secundarias técnicas, telebachilleratos y colegios de bachilleres en comunidades indígenas, pero sin realizar ninguna adaptación a las condiciones socioculturales locales sino simplemente reproduciendo los modelos nacionales (planes y programas, métodos, calendarios, etcétera).

Con estas acciones se ha logrado un incremento histórico de la matrícula en las escuelas oficiales (incluido el subsistema de educación "bilingüe-intercultural”), alcanzándose una cobertura cercana a 100\% en los niveles de la educación básica (primaria y secundaria) en zonas indígenas.

A la par, los gobiernos federal y estatal hicieron declaraciones y acciones en contra de los proyectos autónomos, amenazando con el desmantelamiento de las escuelas, que en algunos casos se llegó a concretar, y expulsando del país al activista norteamericano Peter Brown, líder de la organización "Schools for Chiapas", por considerar que desconoció "con su conducta, a las instituciones de la república encargadas de organizar el sistema educativo nacional" (La Jornada, 26 de julio de 1998). 


\section{$\mathrm{Ni}$ "cambio", ni "esperanza"21}

En las elecciones federales y estatales del año 2000, el pueblo mexicano y chiapaneco manifestó su esperanza en el cambio político, esperanza que fue compartida incluso por los zapatistas, quienes, ante el cambio de gobierno, iniciaron una nueva campaña para promover la elevación a rango constitucional de los Acuerdos de San Andrés. Nuevamente se toparon con el conservadurismo liberal, y el aparato estatal en pleno (legislativo, ejecutivo y judicial federal y estatales) se negó a aprobar la Ley de derechos y cultura indígena y en su lugar aprobó una Ley Indígena incluso más limitada que la que había propuesto el anterior ejecutivo. El EZLN volvió a su resistencia y las acciones en materia educativa continuaron realizándose de forma paralela.

A partir de este momento, el Estado realiza una serie de acciones, principalmente en materia legislativa y en el ámbito de la educación superior y los proyectos independientes se acercan a las instancias oficiales en busca de reconocimiento y de coordinación. Mientras tanto, el EZLN fortalece sus proyectos autónomos y crea instancias de coordinación entre los mismos.

En enero de 2001, a menos de dos meses de haber asumido la presidencia, el ejecutivo federal creó la Coordinación General de Educación Intercultural Bilingüe (CGEIB),

[...] Que tendrá como propósito asegurar que la educación intercultural bilingüe responda con un alto nivel de calidad a las necesidades de la población indígena [...] Promover la participación de las entidades federativas y municipios, así como de los diferentes sectores de la sociedad, pueblos y comunidades indígenas, en el desarrollo de la educación intercultural bilingüe; Promover y asesorar la formulación, implantación y evaluación de programas innovadores de educación intercultural bilingüe [...] (Diario Oficial de la Federación, 22 de enero de 2001: 58).
La CGEIB ha centrado sus esfuerzos en sensibilizar al personal docente de todos los niveles, en el respeto a las expresiones culturales locales, sugiriéndoles estrategias para integrar estas expresiones al momento de abordar ciertos contenidos, pero ajustándose al currículo nacional establecido.

Posteriormente, con la aprobación de la Ley Indígena, se elevó a rango constitucional la obligación del gobierno a:

Garantizar e incrementar los niveles de escolaridad, favoreciendo la educación bilingüe e intercultural, la alfabetización, la conclusión de la educación básica, la capacitación productiva y la educación media superior y superior. Establecer un programa de becas para los estudiantes indígenas en todos los niveles. Definir y desarrollar programas educativos de contenido regional que reconozcan la herencia cultural de sus pueblos, de acuerdo con las leyes en la materia y en consulta con las comunidades indígenas. Impulsar el respeto y conocimiento de las diversas culturas existentes en la nación (Artículo $2^{\circ}$. Constitucional).

También en el artículo 11 de la Ley General de Derechos Lingüísticos de los pueblos indígenas, promulgada en marzo de 2003, se estableció que "las autoridades educativas federales y de las entidades federativas, garantizarán que la población indígena tenga acceso a la educación obligatoria, bilingüe e intercultural [...] Asimismo, en los niveles medio y superior, se fomentará la interculturalidad, el multilingüismo y el respeto a la diversidad y los derechos lingüísticos".

En el ámbito de la educación superior, con recursos del gobierno del estado se creó la Normal Intercultural Jacinto Canek. En el año 2000, la Universidad Pedagógica Nacional (UPN) abrió una maestría en Educación y Diversidad Cultural que opera en las subsedes de San Cristóbal y Palenque y en la sede de Tuxtla Gutiérrez y ofrece una línea de especialización 
$\begin{array}{lll}R & T & A\end{array}$

en educación en contextos interculturales; y en el año 2001, inauguró un diplomado en Educación Intercultural. En la Universidad Autónoma de Chiapas se abrió una maestría en Educación Indígena (en el campus de Humanidades) y, en 2005, una licenciatura en Educación Indígena (en el Instituto de Estudios Indígenas), al tiempo que se crea la Universidad Intercultural de Chiapas. ${ }^{22}$

Por su parte, el nuevo gobierno estatal, que llegó al poder con el apoyo de partidos y organizaciones opositoras, incluidas organizaciones simpatizantes zapatistas y que incorporó a varios líderes sociales en el gabinete, gozaba de un amplio margen de credibilidad entre los activistas políticos de izquierda, credibilidad que se mantuvo tras lograr que el Congreso local votara en contra de la resolución del Congreso federal en torno a los Acuerdos de San Andrés. En las declaraciones de los funcionarios estatales se percibía un espíritu de apertura hacia las propuestas de educación alternativas, incluso hacia los proyectos zapatistas, aunque también se evidenciaba su desconocimiento de las mismas:

El actual gobierno de Chiapas avaló el proyecto de educación autónoma rebelde que grupos de extranjeros han practicado en la zona de operancia del Ejército Zapatista de Liberación Nacional (EZLN).

El secretario de educación Alfredo Palacios Espinosa dijo a los medios de comunicación que el planteamiento del programa educativo zapatista no se contrapone a los objetivos oficiales y aceptó que incluye una fuerte carga ideológica no obstante detalló: "todos los programas educativos contienen ideologías, sólo hay que ser respetuosos de las que ellos sostienen".

El funcionario explicó que al menos 15 mil niños indígenas chiapanecos estudian sus primeros años de escuela (primaria) con el programa "Semillita del Sol" diseñado por el activista americano Peter Brown en los conflictivos municipios de Ocosingo, Las Margaritas y Altamirano donde operan los guerrilleros zapatistas y no se acepta la presencia de profesores del gobierno (http://www.angelfire.com/ab/cambio, marzo del 2001).

Ante esta posición, muchos de los actores que venían trabajando propuestas educativas "independientes" vieron la apertura de un espacio de diálogo y entablaron comunicación con instancias del gobierno con el objetivo de obtener reconocimiento y apoyo oficial para sus propuestas. UNEM vuelve a solicitar el reconocimiento y la implementación de sus propuestas de educación intercultural, tanto de primaria como de bachillerato pedagógico. No encuentra una respuesta definitiva, su solicitud ha sido canalizada hacia diferentes instancias y hasta el día de hoy no ha tenido respuesta para ninguna de sus demandas. Producto de tal indefinición, ha sufrido un adelgazamiento importante que amenaza con su desaparición.

También los integrantes del proyecto ECIDEA se acercaron a la Secretaría de Educación del estado en busca de su reconocimiento. Lo que obtuvieron fueron becas y certificación por parte de PECI, con la promesa de que no interferiría en sus formas de trabajo, pero, ya en la práctica, han tenido que enfrentar algunas restricciones en la contratación de nuevos educadores. Además, el Presidente de la República ha anunciado la intención de hacer reformas a la Ley General de Educación para retirar el registro a todas las escuelas de educación básica que no trabajen con los libros de texto nacionales. ${ }^{23}$

Tras el retorno de los desplazados de Las Abejas a sus comunidades de origen, en el año 2003, el proyecto educativo de esta organización enfrentó una grave crisis, dado que los alumnos regresaron a tomar clases en las escuelas oficiales y se quedó sin población que atender. Aunque se han hecho intentos por mantener el proyecto, estableciendo algunos acuerdos con los profesores oficiales, su futuro es incierto. 
Otros espacios de acercamiento entre proyectos independientes e instituciones gubernamentales han resultado de la participación conjunta en la realización de ciertas acciones, entre las que podemos mencionar el proyecto "tarjetas de autoaprendizaje interculturales bilingües" para la elaboración de material didáctico, en el que participaron UNEM, Las Abejas, ECIDEA y la CGEIB entre otros (proyecto desarrollado entre los años 2001 y 2004), ${ }^{24}$ así como los seminarios y talleres que, sobre educación intercultural, se realizaron entre 1998 y el año 2000, coordinados por CIESAS y UPN, en los que participaron educadores de UnEM, Melel Xojobal y ECIDEA entre otras organizaciones y que desembocaron en la conformación del "Colectivo por una educación intercultural" en febrero del 2001, al que se sumaron ECosur, la Casa de la cultura de San Cristóbal y Guaquitepeq, entre otras.

Posteriormente, el Colectivo promovió y convocó, junto con otras organizaciones e instituciones, a la realización de tres congresos regionales (en Ixtepec, Oaxaca, México, D.F. y Tijuana, Baja California) y un Congreso Nacional de Educación Indígena e Intercultural, en Pátzcuaro, Michoacán, todos durante el año 2002. Dicho congreso convocó "a la sociedad en su conjunto a unir esfuerzos, por una educación nacional intercultural de calidad desde los pueblos y para los pueblos" (La Jornada, 10 de noviembre de 2002). Aunque no ha habido representación de los proyectos zapatistas (han participado ONG, proyectos educativos independientes e instituciones gubernamentales), el Congreso se ha pronunciado por la difusión, reconocimiento y cumplimiento de los Acuerdos de San Andrés.

Sin embargo, son muy pocas las acciones que se han realizado de manera coordinada; más bien, es común encontrar proyectos educativos similares trabajando en los mismos espacios. Situación que, aunque no nueva, se ha visto acentuada, llegando al extremo de que en una pequeña comunidad funcionan al mismo tiempo dos o más escuelas primarias pertenecientes a diferentes proyectos, que se dividen los alumnos en función de su filiación política.

De hecho, los zapatistas, han continuado trabajando de manera autónoma, fortaleciendo y expandiendo todos sus proyectos de educación y avanzando en la coordinación de los mismos. A un año del nacimiento de las Juntas de Buen Gobierno, Gloria Muñoz (2004) hizo un recuento de las acciones en la materia: se capacitaron a más de 300 promotores de educación y se abrieron 42 nuevas escuelas comunitarias en los municipios de la zona "selva fronteriza" (adscritas al Caracol de La Realidad). En los Altos de Chiapas se organizaron los diferentes proyectos de educación primaria, que venían trabajando de manera independiente, creando un modelo único: la Escuela Primaria Rebelde Autónoma Zapatista (EPRAZ), abriéndose 83 nuevas escuelas primarias en septiembre de 2004 (en muchos casos, desplazando a los maestros oficiales), de forma que "en estos momentos, más de cien promotores y promotoras dan clases en igual número de pueblos"; también se dio inicio a un programa de educación para adultos y se continuaron los trabajos en la ESRAZ, coordinándose a través del Sistema Educativo Rebelde Autónomo Zapatista de Liberación Nacional de los Altos de Chiapas (SERAZLN). Por su parte, "en las comunidades de la selva tzeltal cuentan con dos centros de capacitación de promotores de educación, uno recientemente inaugurado [agosto de 2004] en la comunidad La Culebra, en el municipio autónomo Ricardo Flores Magón y otro en La Garrucha [...]". En el territorio del Caracol de Morelia, en el programa educativo Organización para la nueva educación autónoma indígena por la pazy la bumanidad, "un total de 280 delegados de educación imparten clases a 2 mil 500 alumnos de los siete municipios" en más de cien primarias, siete secundarias y un centro de capacitación de promotores; y en la zona Norte, "352 promotores de educación dan clases en 159 escuelas 
en resistencia, de las cuales son totalmente nuevas 37. En ellas se forman cerca de 4 mil niños y niñas zapatistas [...,] funcionan dos centros de capacitación de promotores, uno en Roberto Barrios y otro en Ak'abal $\mathrm{Na}$ " y se trabaja en un proyecto de secundaria". "Lo que sigue, dicen, 'será el sueño de tener nuestra universidad zapatista. Antes, todo esto que tenemos también era un sueño, y mira, ya lo cumplimos".

\section{Reflexiones finales}

Después de todo este recuento, es claro que la dinámica de la educación indígena en Chiapas se modificó radicalmente a partir de 1994 y las acciones zapatistas han constituido un factor fundamental que ha impulsado la implementación de diferentes propuestas con la participación de muchos actores, desde muy diversas perspectivas. La gran variedad de acciones (con sus diferencias y similitudes, con sus rupturas y continuidades) es producto de la complejidad misma del campo educativo: ${ }^{25}$ todas las propuestas han sido implementadas en el marco de las interacciones entre los distintos agentes (colectivos e individuales), en el que ocupan diversas posiciones en torno a ciertas relaciones sociales asimétricas, y a partir de las cuales definen sus acciones (en función también de sus propias trayectorias históricas y de ciertos patrones culturales interiorizados a través del habitus); de lo que resalta el carácter eminentemente político de la educación.

Así es como en estos diez años se incrementó significativamente (a niveles históricos y en tiempos récord) la cobertura de la educación formal en el medio indígena chiapaneco (en todos sus niveles), ampliando considerablemente la oferta educativa para los indígenas y posibilitando el acceso de los mismos mediante una serie de apoyos económicos. Ante la presión social, el Estado mexicano mostró gran capacidad de respuesta en términos cuantitativos; sin embargo, la rigidez de sus estructuras educativas ha hecho prácticamente imposible los cambios cualitativos que permitan avanzar hacia la interculturalidad, por lo que los logros alcanzados implican la extensión intensificada de las prácticas asimilacionistas a la escuela secundaria.

Los avances cualitativos más importantes han sido posibles en las propuestas realizadas al margen del sistema oficial. El EZLN y otras organizaciones independientes han efectuado innovadoras propuestas educativas culturalmente pertinentes (construidas con la amplia participación de los mismos indígenas y en las que la cultura y los conocimientos locales ocupan un espacio primordial), manteniéndose a la vanguardia en este aspecto y enseñándonos que es necesaria una buena dosis de autonomía para hacer viable la interculturalidad educativa.

En cierta forma, los zapatistas han jugado el papel que deben cumplir los movimientos sociales según Ramón Flecha: "las posturas críticas tienen que desarrollar disensos que reconstruyan posteriormente consensos más igualitarios. Ahí es donde está situado el imprescindible papel de los movimientos sociales (Flecha, 1994: 69)" y, como afirma Luis Javier Garrido (2004), los zapatistas de Chiapas siguen mostrando que la utopía está al alcance de la mano, y lo más significativo es que uno de sus mayores logros sea en materia educativa. 
A B I E R T A

\section{Cuadro 1}

Propuestas educativas implementadas en Chiapas 1994-2004

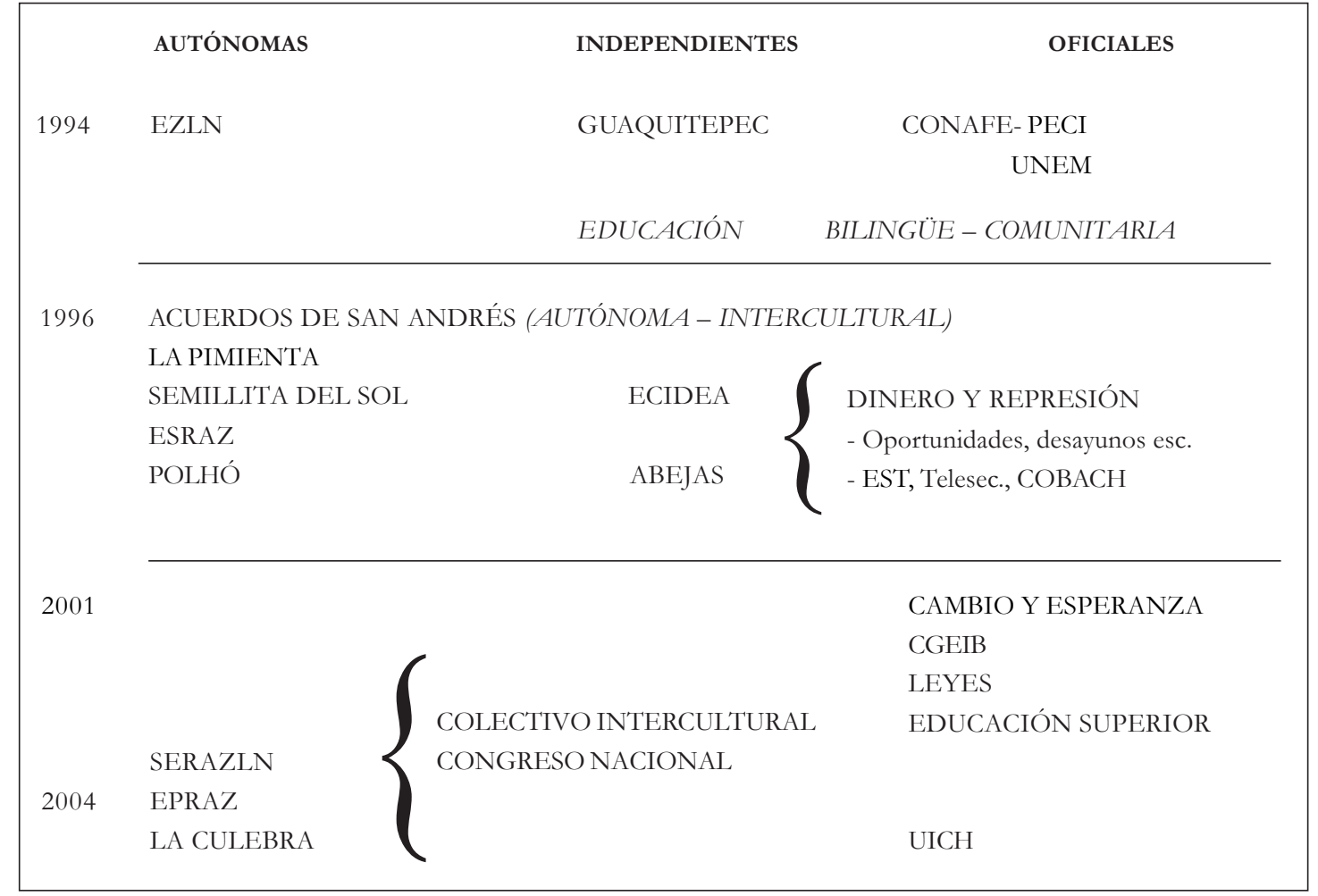

\section{Notas}

${ }^{1}$ Para mayor información sobre la puesta en práctica de algunos de los proyectos abordados en este artículo, véase mi tesis de maestría (Gutiérrez, 2005)

2 Aunque, como veremos más adelante, desde 1990, ya se denominaba oficialmente como "intercultural bilingüe".

${ }^{3}$ Véase Gutiérrez, 2005; González, 2000; Freedson, 1995 entre otros.

${ }^{4}$ Sin lugar a dudas, la lista no abarca todo el universo de propuestas surgidas en estos diez años, pero constituye una muestra representativa de las mismas.

${ }^{5}$ Proceso. Semanario de información y análisis, 21 de febrero de 1994, núm. 903, p. 22.
${ }^{6}$ Antecedente de la modalidad bilingüe-bicultural.

${ }^{7}$ Donde hasta entonces únicamente existían dos escuelas primarias, una bilingüe y una monolingüe, por lo que, en toda la comunidad, únicamente existía una persona con educación secundaria.

8 "Institución privada, no lucrativa, creada en 1972, en la ciudad de México con el propósito de desarrollar modelos alternativos de educación" (Méndez, 2000: 44).

9 "Heterogéneo conjunto de movimientos, asociaciones, comunidades y - posteriormente- instituciones que confluyen en la reivindicación del valor de la "diferencia" étnica y/o cultural así como en la lucha por la pluralización de las sociedades que acogen a dichas comunidades y movimientos" (Dietz, 2003: 13). 
10 "Nominalmente porque dicho tránsito se efectuó a través de un cambio en la denominación utilizada en los documentos correspondientes a la modernización educativa, sin que mediara explicación alguna sobre las razones y los conceptos que fundamentan la adopción de la nueva perspectiva" (Gigante, 2000: 159).

${ }^{11}$ Antonio Muñoz Sedano (2000: 89-92) ha propuesto una clasificación de las propuestas de educación intercultural, que incluye los siguientes grupos: "propuestas o programas dirigidos por una política educativa conservadora", en la que incluye los de tipo asimilacionista que tienden a la desaparición de las culturas minoritarias y su "absorción" en la sociedad nacional, así como los que afirman que los niños de culturas minoritarias aprenden menos por factores biológicos y genéticos; "programas dirigidos por políticas educativas neoliberales", en el que incluye desde los programas compensatorios de tipo paternalista pasando por los biculturales y bilingües, los de promoción del pluralismo cultural hasta los de la unificación europea; y los "programas promovidos desde el paradigma sociocrítico", que proponen "una educación multicultural basada en la identificación de los grupos y problemas sociales para entrar en un proceso de liberación y de conquista de los derechos negados tradicionalmente".

${ }^{12}$ Retomando elementos de varios autores podemos entender la cultura como el entramado de significados que inciden, y sirve como marco interpretativo a las interacciones humanas; construido social e históricamente (a través de procesos de rutinización de la praxis) en el contexto de ciertas relaciones sociales (asimétricas), y que tiene una forma de manifestación subjetivada (emic), en los conocimientos y las representaciones individuales y una forma objetivada (etic), en las prácticas y productos de la actividad humana (rituales, instituciones, formas de organización, tradiciones, símbolos, cosmovisión, vestido, etc.).

${ }^{13}$ Entrevista de B. Petrich y E. Henríquez, La Jornada, México, 6 de febrero de 1994, p. 7.

${ }^{14} \mathrm{El}$ estado de Chiapas fue prácticamente ocupado por el ejército mexicano.

${ }^{15}$ Impulsada como parte de la estrategia de contrainsurgencia aplicada por el Estado mexicano.

${ }^{16}$ Extrañamente sin hacer ninguna mención a los Acuerdos de San Andrés, aun cuando su autor participó en los diálogos.

${ }^{17}$ Una nueva forma de resistencia, ahora pública, a diferencia de las centenarias formas de resistencia indígena (disfrazadas. discretas u ocultas), desarrolladas al nivel de la infrapolitica (véase Scott, 2000).

${ }^{18}$ ONG que "desde 1982 colabora y contribuye al fortalecimiento de las organizaciones sociales campesinas e indígenas en Chiapas y en otros estados del país y que en 1997 establece un equipo regional en Ocosingo" (Lumaltik, 2001: 22).

${ }^{19} \mathrm{Y}$ en algunos casos, con individuos que operaban, de forma personal, desde diverso tipo de instituciones (religiosas, políticas e incluso estatales) y que hicieron posible el acceso a recursos institucionales para la realización de acciones concretas, pero sin un compromiso explícito de las mismas con los proyectos educativos.

${ }^{20}$ Que, por no estar adaptados a la dieta de los indígenas, en buena parte son comercializados a través del mercado local o se utilizan para el consumo de los animales domésticos.

${ }^{21}$ El título del presente apartado hace referencia a los eslóganes de campaña y de gobierno de los grupos que llegaron al poder en el año 2000.

${ }^{22}$ Para mayor información, se pueden consultar sus respectivas páginas de Internet.

${ }^{23}$ Nota publicada en el periódico Cuarto Poder, en abril de 2004.

${ }^{24} \mathrm{El} 31$ de marzo de 2004 presentaron sus resultados ante el gobernador del estado.

${ }^{25}$ Noción acuñada por Pierre Bourdieu y desarrollada en mi tesis de Maestría (Gutiérrez, 2005).

\section{Bibliografía}

Berlanga, Benjamín y Ulises Márquez, 1996, Técnicos en educación rural bilingüe-intercultural (nivel bachillerato). Plan y programas de estudio, Consultores en Educación y Desarrollo S.C., México.

Bertely Busquets, María, 2003, Conflicto intercultural, educación y democracia activa en México. Proyecto de investigación, CIESAS, México.

Bresser Pereira, Luis Carlos y Nuria Cunill Grau, s.a., Lo público no estatal en la reforma del Estado, Paidós, Barcelona.

Diario Oficial de la Federación, 22 de enero de 2001: 58.

Díaz Polanco, Héctor, 2003, Autonomía Regional. La autodeterminación de los pueblos indios, Siglo xxI Editores, $4^{\mathrm{a}}$. Edición, México.

Dietz, Gunther, 2003, Multiculturalismo, interculturalidad y educación: una aproximación antropológica, Universidad de Granada- CIESAS, Granada. 
Enlace Civil, A.C., s.a., Centro de formación para promotores en educación. Semillita del Sol, (http:www.laneta.apc.org/ enlacecivil/educación.htm)

Flecha, Ramón, 1994, "Las nuevas desigualdades educativas" en Castells, Manuel, et al., Nuevas perspectivas críticas en educación, Paidós, España, pp. 55-82.

Freedson-González, Margaret y Elías Pérez Pérez, 1995, "Educación bilingüe-bicultural y modernización en los Altos de Chiapas", América Indígena, vol. LV, núm. 1-2.

Freire, Paulo, 1994, "Educación y participación comunitaria", en Castells, Manuel, et al., Nuevas perspectivas críticas en educación, Paidós, España.

Garrido, Luis Javier, 2004, "La educación zapatista", La Jornada, 24 de septiembre de 2004, México.

Gigante, Elba, 2000, "Una interpretación de la interculturalidad en la escuela" en Díaz Couder, Ernesto y David López Cardeña (coord.), Antología Temática. Educación Intercultural, Secretaría de Educación Pública, México.

González Apodaca, Elena, 2000, Escolarización y etnicidad reinventada: un bachillerato en Santa María Tlabuitoltepec Mixe, Tesis de Maestría, Maestría en Antropología Social CIESAS Occidente, México.

Gobierno Constitucional del Estado de Chiapas, 1993, Educación básica en la Selva. Una experiencia educativa en consolidación, Dirección de Educación Básica, Tuxtla Gutiérrez, Chiapas.

Gutiérrez Narváez, Raúl, 2005, Escuela y zapatismo entre los trotziles: entre la asimilación y la resistencia. Análisis de proyectos de educación básica oficiales y autónomos, Tesis, Maestría en Antropología Social, CIESAs Sureste, México.

Hale, Charles, 2002, "Does multiculturalism menace? Governance, cultural rights and the politics of identifiy in Guatemala", Journal of Latin American Studies, vol. 34, núm. 3.

Klein, Hilary, 2001, “La Educación y La Autonomía Indígena", Centro de Investigaciones Económicas y Políticas de Acción Comunitaria, (www.ciepac.org).

Kymlicka, Will, 1996, Ciudadanía multicultural. Una teoría liberal de los derechos de las minorías, Paidós, Barcelona.

La Jornada, 26 de julio de 1998.

Lumaltik Nopteswanej y Enlace, Comunicación y Capacitación, A.C., 2001, Educándonos para nuestra nueva vida, Ocosingo, Chiapas, mecanuscrito.
McClaren, Peter, 1998, Multiculturalismo revolucionario. Pedagogias de disensión para el nuevo milenio, Siglo xxi Editores, México.

Méndez Guzmán, Alonso, 2000, El modelo educativo del Cesder en la escuela secundaria "Emiliano Zapata" de Guaquitepeq, Chilón, Chiapas, Memoria de práctica profesional, Universidad Autónoma de Chiapas, San Cristóbal de Las Casas, Chiapas.

Muñoz Sedano, Antonio, 2000, "Fundamentación teórica. La educación multicultural e intercultural", en Díaz Couder, Ernesto y David López Cardeña (coord.), Antología Temática. Educación Intercultural, Secretaría de Educación Pública, México.

Muñoz Ramírez, Gloria, 2004, "Chiapas la resistencia”, en Rebeldía, núm. 24, Frente Zapatista de Liberación Nacional, octubre.

Pieck Gochicoa, Enrique, 1996, Función social y significado de la educación comunitaria. Una sociología de la educación no formal, El Colegio Mexiquense-UnICEF, México.

Scott, James C., 1990, Los dominados y el arte de la resistencia. Discursos ocultos, Ediciones Era, México.

Secretaría de Educación, 1996, Reorientaciones 1997, Proyecto Educador Comunitario Indígena, Tuxtla Gutiérrez, Chiapas.

Secretaría de Educación Pública, 1995, Análisis del sistema de educación básica en Chiapas. Una aproximación a partir de indicadores de eficiencia, Delegación especial en Chiapas, Tuxtla Gutiérrez, Chiapas.

Torres, Carlos Alberto, 2001, Democracia, educación y multiculturalismo, Siglo xxi, México.

Unión de Maestros de la Nueva Educación para México (UNEM), 1999, Bacbillerato pedagógico comunitario para la formación de docentes de educación primaria bilingüe intercultural. Plan y programas de estudios, Chiapas, México, mecanuscrito.

Unión de Maestros de la Nueva Educación para México (UNEM), s.a., Modelo curricular de la educación primaria intercultural bilingüe, México, mecanuscrito.

\section{Fuentes de internet}

Global Report, núm. 78, 13-19 julio del 2000, www.agrnews.org

www. bigfoot.com/taspolbe

http:/ / www.angelfire.com/ab/cambio, marzo del 2001 